\title{
Investigating the therapeutic potential of a probiotic in a clinical population with chronic hand dermatitis
}

This article was published in the following Dove Press journal:

Clinical, Cosmetic and Investigational Dermatology

\author{
Wayne P Gulliver' \\ Andrew S Hutton' \\ Noam Ship ${ }^{2}$ \\ 'NewLab Life Sciences Inc., St. \\ John's, NL, Canada; ${ }^{2}$ Research and \\ Development, Bio-K Plus International \\ Inc., Laval, QC, Canada
}

Background: Hand dermatitis or hand eczema (HD) is one of the most common dermatologic conditions. Lesions, scaling, pruritus and pain are chronic and relapsing. Improved $\mathrm{HD}$ has been reported with the probiotic composed of Lactobacillus acidophilus CL1285, Lactobacillus casei LBC80R and Lactobacillus rhamnosus CLR2 (Bio-K+).

Purpose: Investigation of the therapeutic potential of this probiotic as the sole systemic treatment for adults with nonacute HD.

Subjects and methods: A single-center study documented clinical ratings and patient-reported outcomes in adults with chronic HD. The probiotic was taken orally for 12 weeks, adjunctive to standard topical treatments and preventative measures.

Results: Most of the 30 subjects with mild to severe HD were compliant with the probiotic. Around 22 of the 30 subjects were able to complete the study, and of these subjects, an improvement was noted in 19. One required systemic therapy, and one subject was not able to tolerate the probiotic and therefore discontinued the study. $23 \%$ of the subjects achieved clear or almost clear hands by the end of 12 weeks. Pruritus, which was a common complaint at baseline, was improved with $59 \%$ of symptomatic patients within 2 weeks.

Conclusion: It is feasible and safe to administer Bio-K+ for HD. Clinicians saw an improvement in most subjects' hands, and cases of significant improvement in dermatitis were documented. Pruritus was the most rapidly relieved symptom, as reported by patients.

Keywords: hand dermatitis, eczema, CL1285, Lactobacillus, pruritus, Bio-K+

\section{Introduction}

Advances in molecular methods over the past decade have revealed that the human microbiomes of the gastrointestinal tract and the skin are determinants of allergic disease $^{1}$ and skin homeostasis. ${ }^{2}$ Manipulation of these microbial communities is now an emerging treatment axis in dermatology. ${ }^{3-5}$ Compared to other systemic treatments, the relative safety and tolerability of orally ingested probiotic microorganisms makes them attractive for long-term prevention of acute cutaneous states ${ }^{3}$ although their use has outpaced the collection of clinical data. ${ }^{6}$ The expected therapeutic effects for mature illness, even in common conditions, are largely unknown and scarcely documented in a research setting.

Hand dermatitis or hand eczema (HD) is one of the most common dermatological conditions affecting $10 \%$ of the population with dryness, erythema and scaling on the hands coupled with pruritus and pain..$^{7-9}$ Although HD is often the result of multiple exogenous and endogenous causes, a higher incidence of HD is associated with certain
Correspondence: Wayne P Gulliver

NewLab Life Sciences Inc., 187

Lemarchant Rd, St. John's, NL AIC 2H5, Canada

Tel +l 7097535522

Fax +l 7097535478

Email wgulliver@newlabresearch.com 
occupations (occupational dermatitis), such as those involving frequent "wet work" or contact with irritants and allergens (ie, hairdressers, nurses, etc) ${ }^{10-12} \mathrm{HD}$ is a fluctuating condition where hands are transiently or chronically (CHD) affected by lesions. The clinical goals are to prevent progression to an acute clinical state and to manage symptoms, rather than cure the condition. ${ }^{12-14}$ Lesions on the hand are of concern to patients, but intense and constant symptoms of pain and pruritus are a major driver for patients to seek medical help. ${ }^{15}$ Even with proper skin hygiene, acute states of HD can occur and will necessitate potent topical treatments, phototherapy or systemic corticosteroids. ${ }^{11,14}$ These carry an important burden of adverse effects and cannot be used for long term. There is a continued need for tolerable preventative treatments.

Consumers of a probiotic available over the counter in Canada and the United States composed of Lactobacillus acidophilus CL1285, Lactobacillus casei LBC80R and Lactobacillus rhamnosus CLR2, known as Bio-K+ ${ }^{\circledR}(\mathrm{Bio}-\mathrm{K}$ Plus International Inc, Laval, QC, Canada) ${ }^{16}$ have reported improved HD. ${ }^{17}$ The efficacy and safety of this probiotic formulation have been studied quite extensively in diarrhea prevention. ${ }^{18,19}$ To document better and to understand the nature of these improvements, an exploratory, single-center, open-label trial was designed to describe the clinical effects with this probiotic and evaluate its feasibility as the sole systemic treatment for adults with chronic HD, adjunctive to standard topical treatments and preventative measures.

\section{Subjects and methods Study participants}

Thirty subjects were enrolled at one study center in St. John's, Newfoundland and Labrador (NL), Canada, from September 2009 to November 2010. All research procedures performed in the study were in strict accordance with the study protocol, which had full ethics approval from the central ethic committee of the Research Review Board (Waterloo, ON, Canada).

Male and female subjects over 18 years of age with mild to severe HD for $\geq 6$ months and willing to comply with treatment and prevention guidelines ${ }^{12}$ were included in this study. Female patients of childbearing potential were required to have a negative pregnancy test at baseline and used a reliable contraceptive method throughout the study duration.

A dermatologist excluded those affected by skin conditions other than eczema on their hands; had infected lesions on the hands requiring medical treatment, or had known exogenous causes of HD, dyshidrotic or hyperkeratotic eczema. Subjects were not permitted to use systemic treatments for their dermatitis such as a corticosteroid, nonsteroidal anti-inflammatory drug, immunomodulator, biologic (antibodies, fusion proteins, etc), oral primrose oil or traditional Chinese herbal therapies. Subjects were excluded from the study if they had previously received a heart valve replacement, had a serious illness within the 4 weeks preceding the study or presented with a medical condition that in the opinion of the investigator would adversely affect their safe participation in the study or affect the conduct of the study. Also, subjects with a history of substance abuse or a history of poor compliance with medical treatment were excluded.

At screening, a dermatologist performed a thorough examination and completed a checklist of inclusion and exclusion criteria confirming the eligibility of each subject. All eligible subjects received consent forms at least 24 hours prior to their first visit. During their baseline visit, subjects were provided with a full study explanation and answers to any questions before providing their written informed consent to study participation.

\section{Interventions}

Subjects took one enteric-coated capsule daily of a commercially available probiotic containing 30 billion colonyforming units (CFUs) of L. acidophilus CL1285, L. casei LBC80R and L. rhamnosus CLR2 (Bio-K+ ${ }^{\circledR} 30$ Billion; Bio-K Plus International Inc,). Patients were instructed to refrigerate the product and take 1 capsule after breakfast each day for 12 weeks.

At each study visit, the subjects were reminded to follow standard preventative measures to avoid worsening of HD, which includes using a soap-free cleanser; applying a skin moisturizer and avoiding common irritants, harsh cleansers and very hot water. ${ }^{19,20}$ Anti-itch products and over-thecounter hydrocortisone creams were permitted, but the use of systemic treatments for their dermatitis was not permitted.

\section{Outcomes}

The primary outcomes in this study documented the physician- and patient-reported changes in intensity, area affected by irritation and symptoms of pain and pruritus on the hands. The secondary outcomes relate to the feasibility of this treatment regimen in terms of tolerability, safety and the need for higher potency treatments.

\section{Assessments}

Demographic information (ie, age, sex, height, weight, etc) and the HD status were recorded at baseline. To aid recall at each interview, subjects kept a daily diary of their general well-being and any adverse effects. A telephone interview 
was performed at day 7 to ensure compliance with the study protocol and address any questions. The extent and severity of each subject's HD was assessed at baseline and at 2, 4, 8 and 12 weeks of treatment. Subjects who withdrew early were contacted by a study team member to determine the reason for withdrawal and its relatedness to the treatment.

Clinical assessment was performed by one of the two physicians who conducted the study. The Physician's Global Assessment (PGA) of overall HD severity ${ }^{21}$ has been widely used for studies on HD and other types of dermatitis. The PGA considers five states of HD (severe, moderate, mild, almost clear and clear) based on seven observed features (erythema, scaling, hyperkeratosis/lichenification, vesiculation, edema, fissures and pruritus/pain) present on the palm or dorsum of both hands. A state of almost clear or clear at 12 weeks, as a result of a minimum two state changes, was considered a meaningful improvement of HD. The modified Total Lesion Symptom Scale (mTLSS) is a validated tool for $\mathrm{HD}^{22}$ to quantify the seven features of $\operatorname{HD}(0=$ none, $1=$ mild, $2=$ moderate and $3=$ severe) for a maximum score of 21 for each hand. Symptoms of pruritus and pain were scored by a physician based on interview questions with the subject. Some subjects were diagnosed with HD only on one hand. For those subjects, ratings were not collected for the healthy hand.

When subjects reported itchy skin, they further specified the frequency with a 5 -point scale $(1=>10 \%$ of the day, $2=$ only after hand is wet, $3=>30 \%$ of the day, $4=$ most of the time and $5=$ continuously). The presence of pain was reported by subjects using a 5 -point scale $(1=$ strongly disagree to $5=$ strongly agree).

At the end of the 12-week treatment clinicians and subjects reflected on the treatment period. Clinicians estimated the overall percentage improvement for each hand: no change $(0 \%)$, slight (1-25\%), minimal (26-50\%), moderate (51$74 \%$ ) or significant change (75-100\%). Subjects completed a defined schedule of questions related to their current skin condition and its evolution during treatment.

\section{Statistical analyses}

Paired sample $t$-test or repeated measure ANOVA were performed using Statistical Package for the Social Sciences 18.0 at Technical University, Delft, Netherlands, and the Newfoundland and Labrador Centre for Health Information, St. John's, NL, Canada. mTLSS scores were treated as a continuous variable. The testable hypothesis was that the skin condition and burden of symptoms would remain the same overall. The overall treatment effect for each hand was calculated with one-way repeated measures ANOVA. The first analysis tested the effect in per protocol (PP) subjects who had mTLSS data for all 5 time points and excluded individuals with early termination. The second analysis tested the effect in all intention-to-treat subjects using imputed values for early termination, carrying forward the last recorded mTLSS score throughout remainder of trial period. Paired sample $t$-tests tested differences in mTLSS scores between baseline and each time point for each hand.

The evolution of the skin's condition and the presence of symptoms were treated as discrete variables. A significant change in the skin's overall condition meant clinicians had scored a significant change (75-100\%) for each affected hand. Lesions from either hand were considered in reporting the prevalence of lesions and incidence of new lesions. Pruritus was considered frequent if subjects rated itchiness $>30 \%$ of the time on either hand. Pain was considered present if subjects reported pain on either hand. A McNemar's test was performed to detect significant changes in discrete data over time using GraphPad QuickCalcs (GraphPad Software Inc).

\section{Results \\ Baseline characteristics}

A total of 32 patients were screened at the study center and consented to treatment. Two subjects were not included due to a screen failure. Thirty subjects received treatment, and 22 completed the study period of 12 weeks. Most subjects had HD on both hands at baseline; however, four subjects were only affected on one hand (two rights and two lefts).

The majority of the participants were female (68\%), had a history of HD longer than 5 years (87\%) and were experiencing frequent itchiness or painful hands $(83 \%)$. All subjects were in good health with 7 participants $(23 \%)$ reporting a tobacco smoking habit at baseline (mean of 5 packs/week). At the start of the study, 12 participants (40\%) reported regular usage of a moisturizer for HD control (Table 1).

\section{Primary outcomes}

The PGA rated 12 subjects in a better state than baseline $(55 \%)$ and 5 subjects $(23 \%)$ in a clear or almost clear state at the end of the study, representing at least a two state improvement. For the majority of subjects, 19 of 22 (86\%), clinicians estimated a slight, or better, improvement and significant improvement (75-100\% change for each affected hand) in 6 subjects $(27 \%)$. When asked about their hands at the end of the study, about half the subjects reported that their skin condition had improved overall, $10(45 \%)$, or there were no new lesions on either hand, 11 (52\%). Many fewer agreed that their hands were free from irritation, $6(27 \%)$. 
Table I Subject characteristics at baseline, $N=30$

\begin{tabular}{|c|c|c|}
\hline Characteristics & ITT, $\mathbf{N}=\mathbf{3 0}$ & $P P, N=22$ \\
\hline Age & $46( \pm 14)$ & $47( \pm 15)$ \\
\hline Sex, female & $20(67)$ & $15(68)$ \\
\hline History of atopic dermatitis & II (37) & $6(27)$ \\
\hline First outbreak, age & $28( \pm 18)$ & $29( \pm 19)$ \\
\hline First outbreak, age 18 or younger & $9(30)$ & $7(32)$ \\
\hline Hand dermatitis for 5 years or longer & $26(87)$ & $19(86)$ \\
\hline Smoker & $7(23)$ & $5(23)$ \\
\hline Severe (PGA) & $6(20)$ & $5(23)$ \\
\hline Moderate & $17(57)$ & $12(55)$ \\
\hline Mild & $4(13)$ & $3(14)$ \\
\hline Not determined & $3(10)$ & $2(9)$ \\
\hline mTLSS score, right hand & $9( \pm 6)$ & $9( \pm 6)$ \\
\hline mTLSS score, left hand & $9( \pm 5)$ & $9( \pm 6)$ \\
\hline Frequent itchiness ${ }^{\mathrm{a}}$ & $23(77)$ & $17(77)$ \\
\hline Painful hand ${ }^{b}$ & $18(60)$ & $13(59)$ \\
\hline
\end{tabular}

Notes: Data at baseline reported as the mean ( \pm standard deviation) or number of subjects (percentage of total). altchiness on either hand $>30 \%$ of the day. ${ }^{\text {PPresence }}$ of pain on either hand.

Abbreviations: ITT, intention-to-treat; PP, per protocol; PGA, Physician's Global Assessment; mTLSS, modified Total Lesion Symptom Scale.

Although variability of mTLSS scores remained high throughout the study, there was a statistically significant treatment effect of Bio-K+ to reduce the lesion symptom score for each hand in subjects with complete data sets (right hand, $\mathrm{F}=2.8, P=0.027$, left hand, $\mathrm{F}=3.4, P=0.011)$ and when missing data were imputed (right hand, $\mathrm{F}=2.8, P=$ 0.027 , left hand, $\mathrm{F}=3.5, P=0.010$ ). Pairwise comparisons showed that scores were significantly lower than baseline at days 14, 28 and 56, for the right and left hands, but not at day 84 (Table 2).

Frequent itchiness ( $>30 \%$ of the day) was reported by fewer subjects at every follow-up visit, dissipating for 10 of 17 and emerging for 1 in 5 by the end of the study, Figure 1. Painfulness of the hands was also reported in fewer subjects at each visit, dissipating for 10 of 13 and emerging in 2 of 9 by the end of the study.

\section{Secondary outcomes}

A consort flow diagram for this study is shown in Figure 2. Of the 30 subjects who took the probiotic daily, all but one was able to tolerate it. One subject withdrew from the study after 4 weeks because of diarrhea that was probably related to the study medication. One subject experienced a worsening of eczema within the first 2 weeks and withdrew from the study to initiate systemic corticosteroid treatment. Five others withdrew consent before the end of the study, and one was lost to follow-up. The baseline profile of the 22 subjects (73\%) who completed the study PP was not markedly different than the intention-to-treat population (Table 1).

\section{Discussion}

This is one of the first study that is documenting the effects of a probiotic for CHD, a common skin condition. There are numerous studies of probiotics in the treatment of children with atopic dermatitis, $\mathrm{AD}$, and relatively fewer studies in adults. ${ }^{6,23}$ Atopy increases the risk for $\mathrm{HD},{ }^{9,24}$ and about one in three subjects in this study had a history of AD. But subjects with known allergic and exogenous triggers were excluded from this study, delineating a population of commonly seen patients where few treatment options remain.

With no comparator arm, it is not possible to determine if changes were specific to the probiotic treatment. Still, much was learned from this chronic population. First, we could confirm that it was feasible for most of the HD patients to take this probiotic as a capsule once daily within the recommended regimen of preventative measures. Although eight subjects withdrew from the study over the course of 12 weeks, there was no noticeable difference in terms of age,

Table 2 Paired $t$-test for differences in mTLSS scores for each hand

\begin{tabular}{|c|c|c|c|c|c|c|c|c|c|}
\hline & & \multicolumn{4}{|c|}{ Left hand } & \multicolumn{4}{|c|}{ Right hand } \\
\hline & & Mean & $\begin{array}{l}\text { Sample } \\
\text { Size }\end{array}$ & $\begin{array}{l}\text { Mean } \\
\text { difference }\end{array}$ & $P$-value & Mean & $\begin{array}{l}\text { Sample } \\
\text { size }\end{array}$ & $\begin{array}{l}\text { Mean } \\
\text { difference }\end{array}$ & $P$-value \\
\hline \multirow[t]{2}{*}{ Pair I } & Baseline & 9.19 & 26 & 1.88 & 0.015 & 9.15 & 27 & 1.56 & 0.033 \\
\hline & Week 2 & 7.31 & 26 & & & 7.59 & 27 & & \\
\hline \multirow[t]{2}{*}{ Pair 2} & Baseline & 9.54 & 24 & 2.79 & 0.013 & 9.19 & 26 & 2.46 & 0.004 \\
\hline & Week 4 & 6.75 & 24 & & & 6.73 & 26 & & \\
\hline \multirow[t]{2}{*}{ Pair 3} & Baseline & 9.33 & 24 & 2.75 & 0.048 & 9.32 & 25 & 2.80 & 0.015 \\
\hline & Week 6 & 6.58 & 24 & & & 6.52 & 25 & & \\
\hline \multirow[t]{2}{*}{ Pair 4} & Baseline & 9.33 & 21 & 2.57 & 0.098 & 9.09 & 22 & 1.73 & 0.14 \\
\hline & Week 8 & 6.76 & 21 & & & 7.36 & 22 & & \\
\hline
\end{tabular}

Note: $P$-values in bold indicate $P<0.05$.

Abbreviation: mTLSS, modified Total Lesion Symptom Scale. 
20

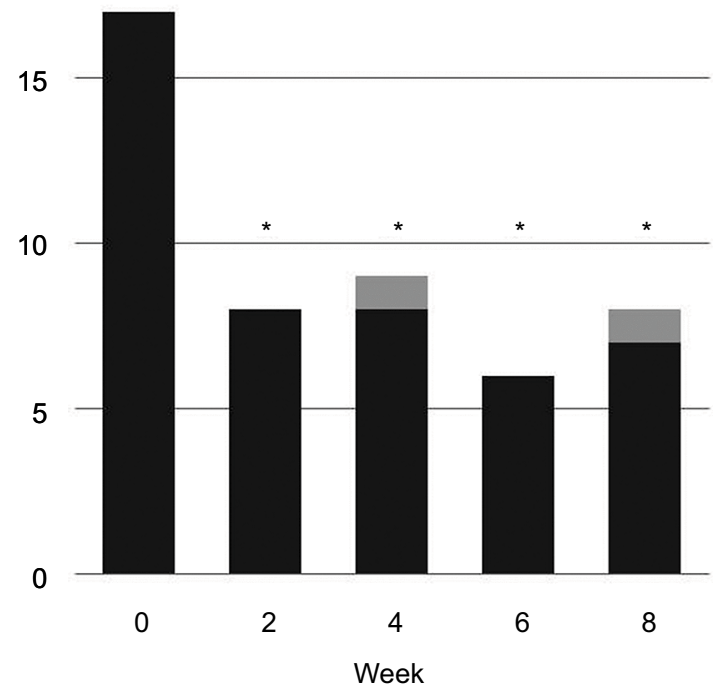

Itchy at baseline

Emergent itchiness
15

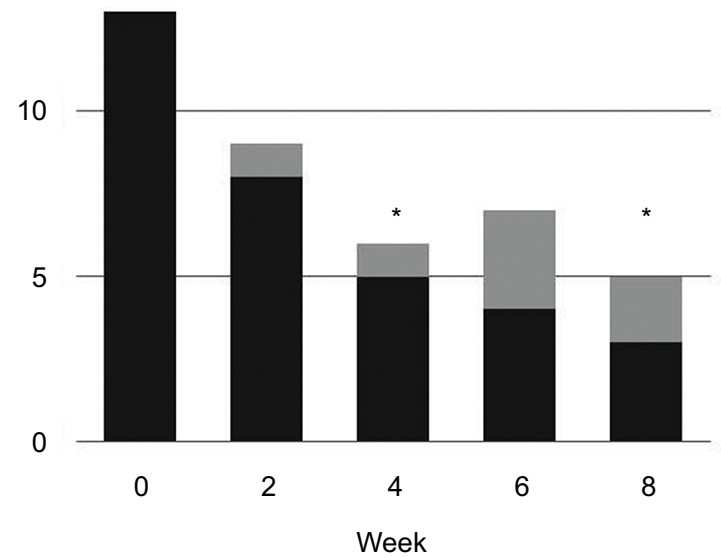

Painful at baseline
Emergent painfulness

Figure I Number of subjects with stable or emergent itchiness ( $>30 \%$ of the time) and painfulness of the hand (per protocol subjects, $N=22$ ). Notes: *McNemar test vs baseline $(\mathrm{N}=22, \mathrm{I} \mathrm{dF})$, two tailed $P<0.05$.

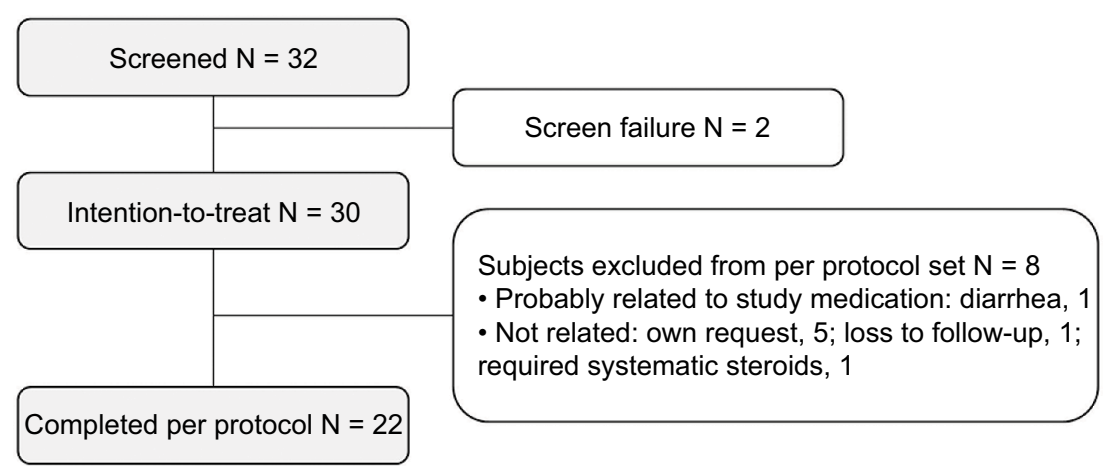

Figure 2 Consort flow diagram.

sex or history of symptom burden between those recruited into the study and those who completed PP. CHD is a condition that waxes and wanes, which can moderate a research participant's motivation. The discontinuation rate $(27 \%)$ was higher than planned but within the expected range for this type of study. A Cochrane review of emollients and moisturizers in eczema looked at a similar patient population who were seeking strategies to avoid acute dermatitis. The incidence of subjects experiencing a flare while taking a moisturizer was $13 \%$, and the likelihood of study discontinuation ranged from $4 \%$ to $25 \% .{ }^{25}$ All but one participant in our study were able to manage their HD without escalating to systemic therapies and thus avoid the adverse effects that come with them. One subject was unable to tolerate the treatment due to gastrointestinal symptoms, which subsided after discontinuation.

The study was designed to explore the evolution of HD symptomatology in a cohort of typical, chronic patients using a variety of dermatology rating instruments. The state of irritation on the hands ranged from mild to severe at the start of the study and fluctuated from month to month. The testable hypothesis for these refractory subjects was that their skin condition and burden of symptoms would remain the same overall. The mTLSS is a precise and validated tool to quantify the affected area, intensity of irritation and symptom burden in HD. ${ }^{22}$ Repeated measures statistical testing of this continuous variable confirmed that there was a significant, albeit small improvement overall. Missing data from subjects 
who discontinued early introduces a potential bias to this type of analysis if they left due to a high symptom burden. To account for this, the analyses were also performed imputing the last observation for missing data but these yielded equivalent results. From a practical point of view, the high variability between subjects and within subjects made this an impractical tool for a small observational study.

As noted in the Canadian Hand Dermatitis Management Guidelines, multiple therapies have been investigated for the treatment of hand eczema. ${ }^{12}$ The efficacy of these ranges from no improvement to improvement up to $70 \%$ using topical steroids. Studies of oral alitretinoin in subjects with severe CHD observed that $48 \%$ of patients achieved a state of clear or almost clear hands at 24 weeks using the PGA, compared to $17 \%$ placebo. ${ }^{22}$ In the physician's impression, most subjects, $86 \%$, in this study had some form of improvement with 12 weeks of treatment, and $23 \%$ attained a state of clear or almost clear rated by the PGA. There was a good agreement between the PGAand patient-reported outcomes. Still, many subjects remained symptomatic and continued to have visible dermatitis. It was not possible to determine which baseline features predicted a meaningful improvement. It could be that Bio-K+ benefited only a subgroup within this diverse population based on endogenous features or their existing intestinal microbiota.

For more than $80 \%$ of subjects with symptoms of frequent itchiness and painful hands at baseline, symptoms dissipated more than would have been predicted by chance and normal distribution. In particular, improvements in itchiness were remarkable. After only 2 weeks of treatment, more than half of the subjects had improved and there were no new emergent cases. Still, there were subjects with frequent itchiness that persisted through treatment, suggesting there could be different etiologies of pruritus or compatibility to treatment. Matsumoto et al saw significant improvements in itchiness symptoms for adult subjects with AD compared to placebo with the probiotic Bifidobacterium animalis subsp lactis LKM512. ${ }^{26}$ In that study, there was a correlation between the production of a bacterial metabolite and kynurenic acid and decreased pruritus. Future studies with Bio-K+ should aim to determine if metabolites in the blood and stool predict symptomatic changes.

Scientific awareness of the role of the gut microbial community in the development and symptomatology of $\mathrm{AD}$ is quite advanced, while the role and function of the skin microbiota is still being elucidated. ${ }^{1,2}$ In theory, the direct application of living microbes to the skin should lead to a more potent response, ${ }^{1}$ yet few clinical cases or interventional studies of topical applications have been reported. ${ }^{2}$ The majority of probiotic studies in dermatology have looked at the oral route of administration, targeting the intestinal tract. ${ }^{3,6,23}$ Although the exact mechanism of probiotics at distant sites is currently unknown, it is likely that the microbes remain in the gastrointestinal tract where they positively influence local inflammation that can translate into systemic effects too., For example, a probiotic formulation containing Lactobacillus salivarius LS-01 improved atopic symptoms in adults and also led to reduced immune reactivity during pollen season. ${ }^{27}$ But the efficacy of probiotics in treating skin conditions, and other medical applications, seems to be strain and dose specific, ${ }^{3}$ and these may even act via differing mechanisms. ${ }^{5}$ Preclinical studies in a murine model of AD hint that Bio-K+ exerts a different systemic effect than corticosteroids. After repeated challenges to oxazolone, topical clobetasol reduced skin inflammation and epidermal proliferation as expected, whereas the effects of orally ingested Bio-K+ were more narrow. ${ }^{28}$ The probiotic resulted in an equivalent reduction in epidermal proliferation, but no other immunological or dermal effects. Although these studies demonstrated that if taken orally this probiotic led to distal changes and altered the pathology of dermatitis differently than topical corticosteroid, we are left with many unanswered questions about the mechanism of action.

\section{Conclusion}

This small study presents an encouraging account in CHD and confirms that it is safe to study Bio- $\mathrm{K}+$ alongside standard preventative measures. Future clinical research should seek to demonstrate an effect of this probiotic above and beyond standard preventative measures and to determine its compatibility with other systemic treatments. The role of the microbiome in skin health is evolving quickly, ${ }^{1,4}$ as is our understanding of how probiotics act at distant sites. ${ }^{1,5}$ A better understanding of the mechanism of action on dermatitis symptoms will help identify which subjects are most likely to benefit from this particular probiotic.

\section{Acknowledgments}

Bio-K Plus International Inc. owns and manufactures the study product $\left(\mathrm{Bio}-\mathrm{K}+{ }^{\circledR}\right)$ and was the sponsor of the study.

\section{Disclosure}

WPG and ASH received research funding as clinical investigators for this study from Bio-K Plus International Inc. NS is an employee of Bio-K Plus International Inc. The authors report no other conflicts of interest in this work. 


\section{References}

1. Huang YJ, Marsland BJ, Bunyavanich S, O'Mahony L, Leung DY, Muraro A, Fleisher TA. The microbiome in allergic disease: current understanding and future opportunities — 2017 PRACTALL document of the American Academy of Allergy, Asthma and Immunology and the European Academy of Allergy and Clinical Immunology. JAllergy Clin Immunol. 2017;139(4):1099-1110.

2. Friedrich AD, Paz ML, Leoni J, González Maglio DH. Message in a bottle: dialog between intestine and skin modulated by probiotics. Int J Mol Sci. 2017;18(6):E1067.

3. Fuchs-Tarlovsky V, Marquez-Barba MF, Sriram K. Probiotics in dermatologic practice. Nutrition. 2016;32(3):289-295.

4. Lynde CW, Andriessen A, Bertucci V, et al. The skin microbiome in atopic dermatitis and its relationship to emollients. J Cutan Med Surg. 2016;20(1):21-28.

5. Reid G, Abrahamsson T, Bailey M, et al. How do probiotics and prebiotics function at distant sites? Benef Microbes. 2017;8(4):521-533.

6. Kim SO, Ah YM, Yu YM, Choi KH, Shin WG, Lee JY. Effects of probiotics for the treatment of atopic dermatitis: a meta-analysis of randomized controlled trials. Ann Allergy Asthma Immunol. 2014;113(2): 217-226.

7. Meding B, Järvholm B. Incidence of hand eczema - a population-based retrospective study. J Invest Dermatol. 2004;122(4):873-877.

8. Diepgen TL, Agner T, Aberer W, et al. Management of chronic hand eczema. Contact Dermatitis. 2007;57(4):203-210.

9. Thyssen JP, Johansen JD, Linneberg A, Menné T. The epidemiology of hand eczema in the general population - prevalence and main findings. Contact Dermatitis. 2010;62(2):75-87.

10. Skoet R, Zachariae R, Agner T. Contact dermatitis and quality of life: a structural review of the literature. Br J Dermatol. 2003;149(3): 452-456.

11. Agner T, Andersen KE, Brandao FM, et al; EECDRG. Hand eczema severity and quality of life: a cross-sectional, multicentre study of hand eczema patients. Contact Dermatitis. 2008;59(1):43-47.

12. Lynde C, Guenther L, Diepgen TL, et al. Canadian hand dermatitis management guidelines. J Cutan Med Surg. 2010;14(6):267-284.

13. Bourke I, Coulson I, English J; British Association of Dermatologists Therapy Guidelines and Audit Subcommittee. Guidelines for the management of contact dermatitis: an update. $\mathrm{Br} J$ Dermatol. 2009;160(5):946-954.

14. Coenraads PJ. Hand eczema. N Engl J Med. 2012;367(19):1829-1837.

15. Van Coevorden AM, Williams HC, Svensson $\AA$, Diepgen TL, Elsner $\mathrm{P}$, Coenraads PJ. Interventions for hand eczema. (Protocol). Cochrane Database Syst Rev. 2002;(3):CD004055.
16. Auclair J, Frappier M, Millette M. Lactobacillus acidophilus CL1285, Lactobacillus casei LBC80R, and Lactobacillus rhamnosus CLR2 (Bio$\mathrm{K}+$ ): characterization, manufacture, mechanisms of action, and quality control of a specific probiotic combination for primary prevention of Clostridium difficile infection. Clin Infect Dis. 2015;60(Suppl 2):S135-S143.

17. Gao XW, Mubasher M, Fang CY, Reifer C, Miller LE. Dose response efficacy of a proprietary probiotic formula of Lactobacillus acidophilus CL1285 and Lactobacillus casei LBC80R for antibiotic-associated diarrhea and Clostridium difficile-associated diarrhea prophylaxis in adult patients. Am J Gastroenterol. 2010;105(7):1636-1641.

18. Goldstein EJC, Johnson SJ, Maziade PJ, Evans CT, Sniffen JC, Millette M, McFarland LV. Probiotics and prevention of Clostridium difficile infection. Anaerobe. 2017;45:114-119.

19. Berndt U, Wigger-Alberti W, Gabard B, Elsner P. Efficacy of a barrier cream and its vehicle as protective measures against occupational irritant contact dermatitis. Contact Dermatitis. 2000;42(2):77-80.

20. English JSC. Occupational dermatoses. In: Burns DA, Breathnach SM, Cox NH, Griffiths CEM, editors. Textbook of Dermatology. 7th ed. Oxford: Blackwell Publishing; 2004:21.8-21.9.

21. Coenraads PJ, van Der Walle H, Thestrup-Pedersen K, et al. Construction and validation of a photographic guide for assessing severity of chronic hand dermatitis. Br J Dermatol. 2005;152(2):296-301.

22. Ruzicka T, Lynde CW, Jemec GB, et al. Efficacy and safety of oral alitretinoin (9-cis retinoic acid) in patients with severe chronic hand eczema refractory to topical corticosteroids: results of a randomized, double-blind, placebo-controlled, multicentre trial. Br J Dermatol. 2008;158(4):808-817.

23. Rather IA, Bajpai VK, Kumar S, Lim J, Paek WK, Park YH. Probiotics and atopic dermatitis: an overview. Front Microbiol. 2016;7:507.

24. Agner T, Aalto-Korte K, Andersen KE, et al. European Environmental and Contact Dermatitis Research Group. Classification of hand eczema. J Eur Acad Dermatol Venereol. 2015;29(12):2417-2422.

25. van Zuuren EJ, Fedorowicz Z, Christensen R, Lavrijsen A, Arents BWM. Emollients and moisturisers for eczema. Cochrane Database Syst Rev. 2017;2:CD012119.

26. Matsumoto M, Ebata T, Hirooka J, et al. Antipruritic effects of the probiotic strain LKM512 in adults with atopic dermatitis. Ann Allergy Asthma Immunol. 2014;113(2):209-216.

27. Drago L, Toscano M, De Vecchi E, Piconi S, Iemoli E. Changing of fecal flora and clinical effect of $L$. salivarius LS01 in adults with atopic dermatitis. J Clin Gastroenterol. 2012;46(Suppl):S56-S63.

28. Ship N, Millette M, Millette E, Boutin Y, Gulliver W. Investigating the therapeutic potential of the probiotic Bio-K+ for hand dermatitis in a clinical population and a mouse model [abstract]. J Int Soc Microbiota. 2017;(4):117. Available from: https://doi.org/10.18143/jism_v4i1. Accessed April 16, 2018.
Clinical, Cosmetic and Investigational Dermatology

\section{Publish your work in this journal}

Clinical, Cosmetic and Investigational Dermatology is an international, peer-reviewed, open access, online journal that focuses on the latest clinical and experimental research in all aspects of skin disease and cosmetic interventions. This journal is included on PubMed. The manuscript management system is completely online and includes a very quick and fair peer-review system, which is all easy to use. Visit http://www.dovepress.com/testimonials.php to read real quotes from published authors 